\title{
The Dark Matter of the Bibliome
}

\author{
David N. Kennedy ${ }^{1}$
}

Published online: 21 October 2015

(C) Springer Science+Business Media New York 2015

Physicists seem to spend a lot of time discussing 'dark matter'. What is dark matter? Dark matter has numerous definitions, such as "Dark matter is a hypothesized form of matter particle that does not reflect or emit electromagnetic radiation. The existence of dark matter is inferred from gravitational effects on visible matter, such as stars and galaxies."1 There are many reasons dark matter consumes this much attention. A significant fraction of the total mass of the universe may be dark, and the forces it exerts have potentially monumental influences in the overall behavior of the universe.

Much closer to home, however, in our neuroscience universe, we too are surrounded by 'dark matter'. Neuroscience dark matter can be thought of as the content of the scientific publication that cannot be searched by typical search engines or human readers. It is dark in the sense that, if it cannot be easily found, it must only be inferred to exist. Surprisingly, there is a vast amount of neuroscientific dark matter. Our usual search engines, such as PUBMED, search over only the MEDLINE $^{2}$ indexed parts of a publication (author, abstract, keywords, etc.), but not any of the elements of the full text of an article. Google Scholar ${ }^{3}$ searches deeper into the text of publications, but the basic building blocks of the neuroscientific observation are still hidden to these searches. This is because most of these elemental observations are embedded

\footnotetext{
${ }^{1}$ http://physics.about.com/od/glossary/g/darkmatter.htm.

${ }^{2}$ https://www.nlm.nih.gov/bsd/pmresources.html.

${ }^{3} \mathrm{https}: / /$ scholar.google.com/.
}

David N. Kennedy

David.Kennedy@umassmed.edu

1 Department of Psychiatry, University of Massachusetts Medical School, Worcester, MA, USA into the figures and tables (as well as sometimes idiosyncratic free text) that defy simple decoding.

Neuroscience dark matter is 'dark' because it lacks an a priori specification for the information content structure. MEDLINE provides a clear structure for how article content is expressed. ${ }^{4}$ Publications, as a whole, have an accepted 'standard' for how articles are structured (methods, results, discussion, etc.). Free text is even bound by the grammatical and syntactic constructs of the language that aid in the decoding of textual passages. More often than not, however, there is no standardized information specification that accompanies the figures and tables of an article. Captions and headings and embedded text (as well as substantial domain knowledge) are what make the intrinsic content of these objects intuitive to the human reader, but dark to the computerized search. Hence the key to accessing this information is very domain specific, and the breadth of domains covered in neuroscience is expansive.

Numerous efforts exist that attempt to enlighten these dark parts of the published literature. These efforts typically are highly domain specific, and linked with a databasing effort designed to capture a specific set of observation instances. Most often, these efforts require human expert curation and operate in the post-publication (pdf or html) interpretation of the published literature. In the neuroimaging domain, BrainMap Database ${ }^{5}$ and $\operatorname{SuMSDB}^{6}$ capture 'activation' foci from publications of voxel-based analysis of structure or function in humans. The Internet Brain Volume Database (IBVD) ${ }^{7}$ captures reports of neuroanatomic structural volumetric

\footnotetext{
${ }^{4}$ Kennedy, D.N. (2013). Data citation and the author byline: who's line is it anyway? Neuroinformatics, 11(3),263-6.

${ }^{5} \mathrm{http} / / /$ www.brainmap.org/.

${ }^{6} \mathrm{http}: / /$ sumsdb.wustl.edu:8081/sums/index.jsp.

${ }^{7}$ Kennedy, D.N., Hodge, S.M., Gao, Y., Frazier, J.A., Haselgrove, C. (2012). The internet brain volume database: a public resource for storage and retrieval of volumetric data. Neuroinformatics, 10(2), 129-40.
} 
observations. While the utility of the resultant databases in these cases is clear, they are perpetually behind the rate and content of the publication and are very costly to keep up to date.

There are hybrid initiatives that, in part, depend on natural language processing (NLP), but then focus attention on a specific domain; the specific domain holds the key to an expectation of the types of content that will be presented in the tables and figures of the article. An example of this is Neurosynth. ${ }^{8}$ Neurosynth automatically extracts coordinate tables and keywords from the available HTML versions of specifically covered journals. From this, the likelihood of association of brain locations and functional terms can be computed. There are great benefits in that NLP can be applied to a large corpus of full text. Limitations to this type of approach, however, exist in establishing the detailed associations between interpretation statements (in the body of an article) and the specific supporting data (of a particular table or figure).

These examples demonstrate that we, as an information field, are not yet where we need to be to make the routine connection between the interpretation we make in an article and the data that supports it. With the rising concerns over the reproducibility of science, ${ }^{9,10}$ this connection is an integral part of moving the field forward.

Exposing the 'dark' content of the research article is essential to advancing the cause of reproducibility. Readers of this journal, long accustomed to the obligatory Information Sharing Statement ${ }^{11}$ (that was an initial attempt at explicitly exposing the tools and data necessary for an article in the journal), may have recently noticed the RRID identifiers being used in the methods and Information Sharing Statements. The Research Resource IDentifier effort, ${ }^{12}$ spearheaded by the Neuroscience Information Framework, ${ }^{13}$ is an explicit attempt to create a proper accounting and enumeration of the research resources (tools, animal strains, antibodies, etc.) that are used in a publication (in contrast to arbitrary and non-specific literature citation or antiquated manufacturer/location attribution). This type of explicit declaration is just the tip of the iceberg for expressing precise detail in the context of the running narrative of the scientific publication.

The field can go farther in this direction of detailed content exposure. The field can move faster in adoption of procedures that increase the reproducibility of research findings. It takes will power and commitment from the investigators, and

\footnotetext{
${ }^{8} \mathrm{http}: / /$ www.neurosynth.org/.

${ }^{9}$ Nuzzo, R. (2015). How scientists fool themselves - and how they can stop. Nature, 526(7572), 182-5.

${ }^{10}$ Ioannidis, J.P. (2005). Why most published research findings are false. PLoS Medicine, 2(8), e124.

${ }^{11}$ Kennedy, D.N. (2004). Barriers to the socialization of information. Neuroinformatics, 2(4), 367-8.

$12 \mathrm{http}: / /$ f1000research.com/articles/4-134/v1.

${ }^{13} \mathrm{http}: / /$ neuinfo.org/.
}

perhaps some prodding by journals and funders. It also takes standards, infrastructure and resources that serve the community to enable adoption of the best practices for reproducible science.

It is time to expand the engagement of the author in the data-to-result connection. Authors already do a good job of citing references and complying with PubMed Central requirements. Expanded compliance in the use of existing explicit resource (data, software, etc.) utilization reporting will be helpful, but deeper data-result linkage will also require additional author effort. Elaborating upon the success of the RRID pilot program, the NIF is soon to embark on an authorin-the-loop pilot to study the practicalities of prospective annotation of human brain coordinate tables, as generated from functional MRI (fMRI) or voxel-based morphometry (VBM) ${ }^{14}$ As this is an area where there has already been some progress in automated text recognition, additional expert markup will be valuable in further tuning these automated approaches. This approach targets the period in time where authors are deeply engaged in the publication process, the time between acceptance of the article and final page proof acceptance. It is in this time period, with a modest commitment by the author of 15 minutes or less, that seems ripe for extracting additional content markup. As these markup systems become more efficient, the ability of the author to annotate more deeply becomes available. Thus there is a shared commitment to the process: the author to engage in annotation, the neuroinformaticists to make tools for annotation more efficient, and the publications to enforce annotation and utilization of the public resources that host this deep content. This particular coordinate markup exercise relies on the existence of standards and resources. The Montreal Neurological Institute (MNI) 152 coordinate system ${ }^{15}$ (a derivative of the Talairach coordinate system ${ }^{16}$ ) is the standard coordinate system used to represent the human brain activation type of data. The Cognitive Atlas, ${ }^{17}$ the Cognitive Paradigm Ontology ${ }^{18}$ and the BrainMap Behavioral Domains ${ }^{19}$ are standards that are available to express the nature of the fMRI experiment. Resources exist to host these data representations, including BrainSpell, ${ }^{20}$ Neurosynth $^{21}$ and

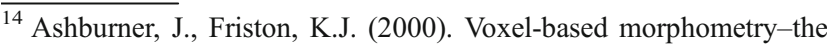
methods. Neuroimage, 11(6 Pt 1), 805-21.

${ }^{15}$ Evans, A.C., Janke, A.L., Collins, D.L., Baillet, S. (2012). Brain templates and atlases. Neuroimage, 62(2), 911-22.

${ }^{16}$ Talairach, J., Tournoux, P. (1988). Co-planar stereotaxic atlas of the human brain. New York: Thieme Medical Publishers, Inc.

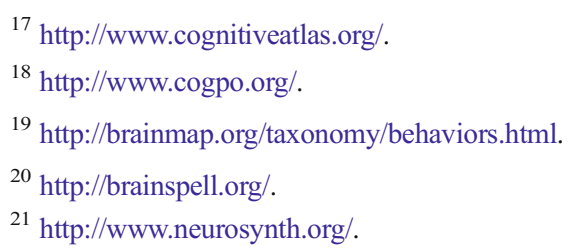

${ }^{17} \mathrm{http} / / / \mathrm{www} . \operatorname{cog}$ nitiveatlas.org/.

$18 \mathrm{http}: / / w w w . c o g p o . o r g /$.

${ }^{19} \mathrm{http}$ //brainmap.org/taxonomy/behaviors.html.

${ }^{20}$ http://brainspell.org/.

${ }^{21} \mathrm{http}: / /$ www.neurosynth.org/.
} 
Neurovault. ${ }^{22}$ Emerging standards for the NeuroImaging Data Model (NIDM), ${ }^{23}$ that is being adopted by many of the standard neuroimaging tools, such as SPM, ${ }^{24} \mathrm{FSL},{ }^{25} \mathrm{AFNI},{ }^{26}$ etc. enables this annotation to be generated by the software itself, greatly reducing the time and effort needed by the author for this markup.
As a community, we must begin to embrace the idea that reproducibility of an observation is as important as novelty; and that exposing all critical details of a research observation is as important as the conclusion (or its 'impact factor'). Doing so is critical to moving the field forward more efficiently, as opposed to spending vast resources chasing irreproducible results.

$\overline{22}$ http://www.neurovault.org/.

${ }^{23}$ Keator, D.B., Helmer, K., Steffener, J., Turner, J.A., Van Erp, T.G., Gadde, S., Ashish, N., Burns, G.A., Nichols, B.N. (2013). Towards structured sharing of raw and derived neuroimaging data across existing resources. Neuroimage, 82, 647-61.

${ }^{24} \mathrm{http}: / /$ www.fil.ion.ucl.ac.uk/spm/.

${ }^{25} \mathrm{http} / / /$ fsl.fmrib.ox.ac.uk/fsi/.

${ }^{26}$ http://afni.nimh.nih.gov/afni. 